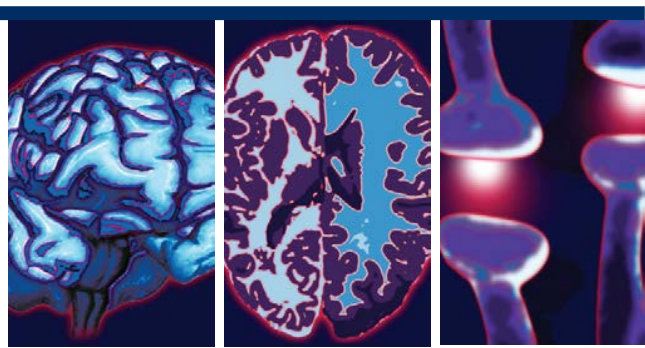

\title{
The Pre-Existing Depressive Disorders, Substance Use Disorders Predicted the Suicidal Death of the Patients with Eating Disorders - A Preliminary Result of National Health Insurance Research Databases in a Chinese Population
}

\author{
Chiu-Lan Yan ${ }^{1,2,3}$, Jung-Chen Chang ${ }^{4,5}$, Shu-Chuan Weng ${ }^{6}$, Ming-Kung Yeh" ${ }^{3, *}$, Chin-Bin Yeh ${ }^{1,7, *,+}$
}

\begin{abstract}
Objective

Recent evidence suggests that additional psychiatric disorders in patients with eating disorders (ED) may contribute to suicide risk. The aim of our study was to investigate the association between eating disorders, its comorbidity and suicidal deaths by the analyzing the data from National Health Insurance Research Databases (NHIRD) and statistical reports on the causes of death.
\end{abstract}

\section{Methods}

We identified 19,648 patients with ED diagnoses from the Taiwan National Health Insurance Database between 2001 and 2012. The patients who had been diagnosed eating disorders $\geq 4$ times at outpatient visits, or had $\geq 1$ time of hospitalization were recruited as the group of $\mathrm{ED}(\mathrm{N}=9974)$. The outcome of measurements was defined as the death of the group of eating disorders either by suicide $(\mathrm{N}=113)$, accidental death $(\mathrm{N}=35)$ or others $(\mathrm{N}=97)$. Cox regression was applied to investigate the relationship between psychiatry comorbidities and the suicidal death of the patients with ED.

\section{Results}

Age of onset with Anorexia Nervosa (AN) was significantly younger than Bulimia Nervosa (BN) or Eating disorders, NOS (EDNOS) in patients with ED. The risk of suicide had 2.4-fold higher of preexisting (aHR=2.4; 95\% $\mathrm{Cl}=1.6-3.7$ ) but not concurrent ( $\mathrm{aHR}=1.0 ; 95 \% \mathrm{Cl}=0.7-1.5$ ) or subsequent (aHR $=0.8 ; 95 \% \mathrm{Cl}=0.4-1.3)$ any psychiatric comorbidity with $\mathrm{ED}$ patients than without psychiatric comorbidity. The risk of suicide was 1.8 -fold higher in patients with previous major depressive disorder (aHR=1.8; 95\% Cl=1.2-2.7), and 2.6-fold higher in patients with prior substance use disorder (aHR=2.6; $95 \% \mathrm{Cl}=1.5-4.5$ ). Patients with four or more psychiatric comorbidities had a 6.3fold increased risk of suicide (aHR=6.3; $95 \% \mathrm{Cl}=3.1-12.8 ; \mathrm{p}<0.05)$.

\footnotetext{
'Graduate Institute of Medical Sciences, National Defense Medical Center, Taipei, Taiwan

2Department of Pharmacy Practice, Tri-Service General Hospital, National Defense Medical Center, Taipei, Taiwan

${ }^{3}$ School of Pharmacy, National Defense Medical Center, Taipei, Taiwan.

${ }^{4}$ School of Nursing, College of Medicine, National Taiwan University, Taipei, Taiwan

${ }^{5}$ Department of Nursing, National Taiwan University Hospital, Taipei, Taiwan

${ }^{6}$ Bachelor's Degree Program of Golden-Age Well-being Management, Yuanpei University of Medical Technology, Hsinchu, Taiwan

${ }^{7}$ Department of Psychiatry, Tri-Service General Hospital, School of Medicine, National Defense Medical Center, Taipei, Taiwan

${ }^{\dagger}$ Author for correspondence: Chin-Bin Yeh, PhD, Department of Psychiatry, Tri-Service General Hospital, National Defense Medical

Center, 325, Sec. 2, Cheng-Gong Road, Nei-Hu District, Taipei 114, Taiwan, Republic of China, Tel: +886-915-376-404; Fax: +886-2-

87927154; e-mail: chinbinyeh@gmail.com

*These authors contributed equally to the research work.
} 


\section{Conclusion}

The previous psychiatric comorbidities including major depressive disorder and substance use disorder were associated with the suicidal death in patients with ED. The patients with more psychiatric comorbidities had a higher risk of suicide.

\section{Keywords}

Suicide, Mood disorder, Eating disorders, Anorexia nervosa, Bulimia nervosa, Health insurance database.

\begin{abstract}
Introduction
Eating disorders (ED) are complex chronic mental disorders, accompanied abnormal eating habits and weight control behavior. ED are relatively rare among the general population, but its affect adolescent in many developed countries [1]. In the $20^{\text {th }}$ century, ED attitudes and behaviors increased dramatically across Asia's high-income populations of young females [2], and psychiatric disorders associated with risk for the development of ED [3]. Previous studies have individuals with $\mathrm{ED}$ report histories of anxiety [4], depression [5], substance abuse [6], and personality disorders [7] maybe play an essential role in the development of eating disorders.
\end{abstract}

However, Clinical studies also indicated that comorbid psychiatric disorders had been associated with suicide attempts in individuals who have an ED [8-12]. As such as the rate of suicide attempts is $3-20 \%$ and $25-35 \%$ in patients with anorexia nervosa and bulimia nervosa, respectively [9], but little is known about the risk factors associated with suicidal death among the patients with ED. In addition, although most studies have used community samples or enrolled patients at primary care clinics $[13,14]$, even in Asia have been cross-sectional survey questionnaires with face-to face interviews or case reports in select population [15-18], the results of the studies were inconsistent due to the small sample sizes, racial differences, and the use of different assessment scales and changes in the sources of diagnostic criteria. Here we present an investigation with regards to the impact of the characteristics including the subtypes of $\mathrm{ED}$, demography data, and psychiatric comorbidities in the relationship between ED and suicidal death with the analysis of Databases.

\section{Methods}

\section{- Data source}

All data had been obtained from the Taiwan's Health and Welfare Data Science Center,
Ministry of Health and Welfare between 2011 and 2012. Data includes the National Health Insurance Database, statistics reports on the causes of death, and household information, regarding annual national marital status and level of education. The data transmissions were encrypted and linked with a personal ID between Databases. The Taiwan National Health Insurance scheme covers $99 \%$ of Taiwan's population and provides data on consultation, emergency consultation, and inpatient diagnoses of insured people. This database demonstrates the healthcare utilization of Taiwanese citizens [19].

\section{- Study population}

We identified the ED group based on an International Classification of Diseases, Ninth Revision, Clinical Modification (ICD-9-CM) code $307.5 \mathrm{X}$ and 307.1 of the National Health Insurance Database (NHID) from 2001 to 2012. There were 19,648 patients were screened with the diagnosis of eating disorders by psychiatrists in NHID. The ED group was defined as either having been diagnosed ED equal to or more than four times at outpatient visits or had at least one time of hospitalization with the diagnosis of eating disorders $(n=9,974)$. They were included in the current study and classified into the four following groups based on the diagnosis: AN (anorexia nervosa, ICD-9-CM code 307.1), BN (bulimia nervosa, ICD-9-CM code 307.51), AN+BN (the presence of both anorexia nervosa and bulimia nervosa, ICD-9-CM code 307.1 and 307.51), and EDNOS (eating disorders not otherwise specified, ICD-9-CM code 307.52-307.59). The followup period was defined from the time of first ED diagnosis until death or study termination. Figure 1 outlines the process of sample inclusion.

\section{- Potential confounders}

In this study we identified psychiatric comorbidities including anxiety disorders (ICD9-CM code 300 except 300.4), major depressive disorders (ICD-9-CM code 296.2, 296.3, 296.82, 300.4), bipolar disorders (ICD-9-CM 
The Pre-Existing Depressive Disorders, Substance Use Disorders Predicted the Suicidal Death of the Patients with Eating Disorders - A Preliminary Result of National Health Insurance Research Databases in a Chinese Population

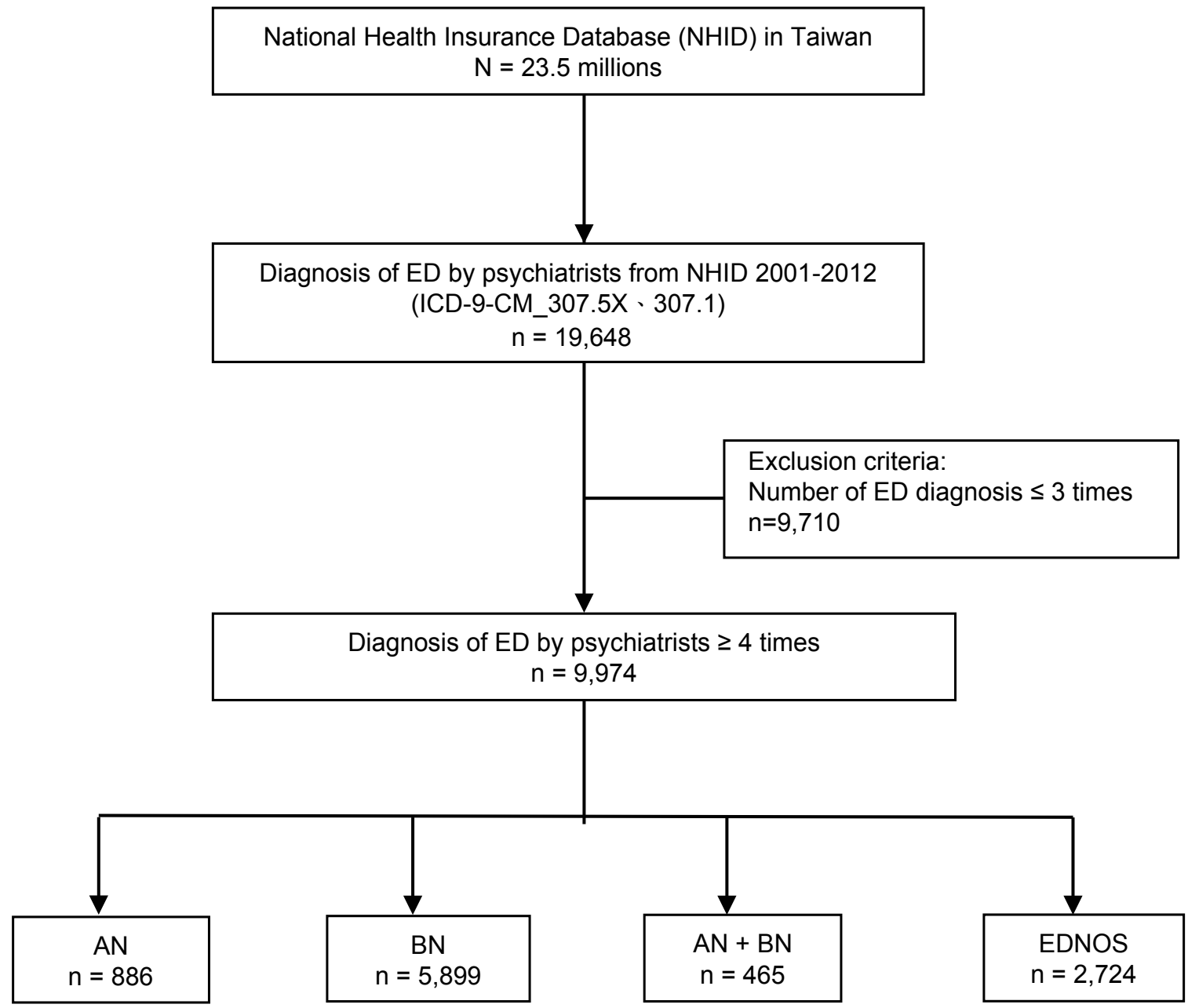

Figure 1: Flow chart of case enrollment from the National Health Insurance Database in Taiwan.

ED: Eating Disorder; AN: Anorexia Nervosa; BN: Bulimia Nervosa; AN+BN: Anorexia Nervosa and Bulimia Nervosa; EDNOS: Eating Disorder not Otherwise Specified.

code 296.0, 296.1, 296.4, 296.5, 296.6, 296.7, 296.80, 296.81, 296.89, 296.90), schizophrenia (ICD-9-CM code 295), substance-use disorders (ICD-9-CM code 291, 292, 303, 304, 305), and personality disorders (ICD-9-CM code 301) of the patients with ED. Information on the comorbidities mentioned above was obtained with the ICD-9-CM codes occurring at 4 or more times during medical visits.

We also used the following parameters included income group, treatment period (time of the first ED diagnosis and treatment, to the time of the last ED diagnosis), history of suicide (E950-959) and information regarding the marital status and educational level were collected from the connected household data. The status of death (including a cause of death/suicide/unnatural death) of all follow-up cases of ED was collected from mortality statistics. The occurrence sequence of first diagnose psychiatric comorbidities were defined pre-existing (the period before ED), concurrent (in the same period with ED), and subsequent (the period after ED).

\section{n Statistical analysis}

The data analyzed in this study included gender, marital status, educational level, comorbidity, which were presented as percentages, while age at the time of treatment, treatment period, length of the follow-up, were presented as mean \pm standard deviation. The differences of all variables between the different types of ED were evaluated using Chi-squared tests or a single-factor analysis of variance (ANOVA). The Cox proportional hazard model was used to investigate the association of suicide risk and mental illness in patients with ED. Statistical significance was set at $P<0.05$. All data were analyzed using the statistical software, SAS Version 9.3 (SAS Institute, Inc, 1995, Cary, NC). 


\section{Results}

\section{- Preliminary analyses}

In this study, we included 9,974 ED patients that were separated into four subtypes. The four subtypes were included as follows: 886 AN (8.9\%), 5899 BN (59.1\%), $465 \mathrm{AN}+\mathrm{BN}$ (4.7\%) and 2724 EDNOS (27.3\%) and the age of onset about $23.0 \pm 10.4$ years, $29.1 \pm 10.4$ years, $22.5 \pm 6.01$ years and $30.4 \pm 10.9$ years in $\mathrm{AN}, \mathrm{BN}, \mathrm{AN}+\mathrm{BN}$ and EDNOS, respectively. While AN is the most common age was 11-20, another subtype is most common among aged 21-30. The average duration of treatment about two years, but $\mathrm{AN}+\mathrm{BN}$ was longest treatment ( $4.4 \pm 3.3$ years). $56.7 \%$ of the $\mathrm{BN}$ patients reported at death by suicidal was the highest in all group, while accidental deaths was the highest in the $\mathrm{AN}+\mathrm{BN}$ patients $(26.7 \%$ of all deaths in the group). The demographic characteristics of four subtype ED in Table 1.

\section{- The risk factors associated with suicide}

The majority of ED patients were female (91.7\%) and single $(78.8 \%)$. As showed in Table 2, the risk of suicide in patients with AN was 2.2-fold higher than that in patients with other ED groups $(\mathrm{aHR}=2.2 ; 95 \%$ confidence interval $[\mathrm{CI}]=1.2-$ 4.3). Patients with a history of suicide attempted had 6.1-fold higher suicidal risk $(\mathrm{aHR}=6.1 ; 95 \%$ $\mathrm{CI}=3.5-10.8)$ compared without such a history. The risk of suicide had 2.4-fold higher of preexisting of any psychiatric comorbidity with ED patients than without psychiatric comorbidity $(\mathrm{aHR}=2.4 ; \quad 95 \% \quad \mathrm{CI}=1.6-3.7)$. However, concurrent (in the same period with ED) and subsequent (the period after ED) psychiatric comorbidities were not significantly associated with risk of suicide for ED patients. Table 3 also reveals that the risk of suicide was 1.8 -fold higher in patients with comorbid pre-existing depression $(\mathrm{aHR}=1.8 ; 95 \% \mathrm{CI}=1.2-2.7)$, and 2.6-fold higher in patients with comorbid prior substance use disorder $(\mathrm{aHR}=2.6 ; 95 \% \mathrm{CI}=1.5$ 4.5). In addition, we found that patients with 4 or more pre-existing psychiatric comorbidities had a 6.3-fold increased risk of suicide $(\mathrm{aHR}=6.3$; 95\% CI=3.1-12.8; $\mathrm{p}<0.05$ ).

\section{Discussion}

Consistent with previous studies [20,21], our result indicated that more female patients than male patients with ED diagnose. Patients with AN were found to have an earlier onset of disease aged $11-20$ years $(n=429,48.4 \%)$, while patients in the other three ED groups were mostly 21-30 years. Our finding is similar to the results of an early investigation of ED from United States National database of Health insurance [22,23], although gender and age did not influence risk factor of suicide with ED.

Our finding indicated that history of major depressive disorder, but not concurrent depressive disorders predicted suicide of the patients with ED. However, the concurrent rate with depression (9.4-21.2\%) had been found to be higher than those who without (1$3 \%$ ) of patients with $\mathrm{BN}$ in previous studies [24,25]. Godart et al. [26] also found the

\begin{tabular}{|c|c|c|c|c|c|}
\hline \multirow{2}{*}{ Sub-groups $(n=9974)$} & AN & BN & AN+BN & EDNOS & \multirow[b]{3}{*}{$P$ value } \\
\hline & $(n=886)$ & $(n=5899)$ & $(n=465)$ & $(n=2724)$ & \\
\hline Variable & \multicolumn{4}{|c|}{ n (\%) or mean \pm S.D. } & \\
\hline Age of onset (years) & $23.0 \pm 10.4$ & $29.1 \pm 9.3$ & $22.5 \pm 6.01$ & $30.4 \pm 10.9$ & $<0.001$ \\
\hline$\leq 10$ & $15(1.7)$ & $7(0.1)$ & 0 & $44(1.6)$ & \multirow{6}{*}{$<0.001$} \\
\hline $11-20$ & $429(48.4)$ & $926(15.7)$ & $196(42.2)$ & $401(14.7)$ & \\
\hline $21-30$ & $291(32.8)$ & 2792 (47.3) & $232(49.9)$ & 1088 (39.9) & \\
\hline $31-40$ & $106(12.0)$ & $1423(24.1)$ & $27(5.8)$ & $716(26.3)$ & \\
\hline $41-50$ & $25(2.8)$ & $602(10.2)$ & $\#$ & $338(12.4)$ & \\
\hline$>50$ & $20(2.3)$ & $149(2.5)$ & \# & $137(5.0)$ & \\
\hline Duration of treatment (years) & $2.0 \pm 2.4$ & $2.3 \pm 2.5$ & $4.4 \pm 3.3$ & $1.7 \pm 1.9$ & $<0.001$ \\
\hline Study Follow-up (years) & $6.5 \pm 3.8$ & $6.1 \pm 3.6$ & $7.9 \pm 3.7$ & $5.5 \pm 3.6$ & $<0.001$ \\
\hline All causes of death & $42(4.7)$ & $127(2.2)$ & $15(3.2)$ & $61(2.2)$ & $<0.001$ \\
\hline Suicide & $13(31.0)$ & $72(56.7)$ & $6(40)$ & $22(36.1)$ & \multirow{3}{*}{0.004} \\
\hline Accidental death & $4(9.5)$ & $19(15.0)$ & $4(26.7)$ & $8(13.1)$ & \\
\hline Other causes & $25(59.5)$ & $36(28.3)$ & $5(33.3)$ & $31(50.8)$ & \\
\hline
\end{tabular}


The Pre-Existing Depressive Disorders, Substance Use Disorders Predicted the Suicidal Death of the Patients with Eating Disorders - A Preliminary Result of National Health Insurance Research Databases in a Chinese

Population

\begin{tabular}{|c|c|c|c|c|}
\hline \multirow[b]{2}{*}{ Variable } & \multicolumn{4}{|c|}{ ED patient $(n=9974)$} \\
\hline & $\begin{array}{l}\text { n (\%) } \\
\text { or mean } \pm \text { S.D. }\end{array}$ & HR (95\% Cl) & $\operatorname{aHR}(95 \% \mathrm{CI})$ & $P$ value \\
\hline \multicolumn{5}{|l|}{ ED Disorder } \\
\hline Other ED & $2724(27.3)$ & 1 & 1 & \\
\hline AN & $886(8.9)$ & $1.8(1.0-3.4)$ & $2.2(1.2-4.3)$ & 0.01 \\
\hline BN & $5899(59.1)$ & $1.4(0.9-2.2)$ & $1.4(0.9-2.2)$ & 0.15 \\
\hline$A N+B N$ & $465(4.7)$ & $1.1(0.5-2.7)$ & $1.2(0.5-3.1)$ & 0.66 \\
\hline Age (in y/o) & $28.6 \pm 10.0)$ & $1.0(0.9-1.0)$ & $1.0(0.9-1.0)$ & 0.4 \\
\hline Low-income & $486(4.9)$ & $0.3(0.1-1.4)$ & $0.3(0.8-1.1)$ & 0.07 \\
\hline Female gender & 9148 (91.7) & $1.3(0.6-2.6)$ & $1.0(0.5-2.1)$ & 0.97 \\
\hline \multicolumn{5}{|l|}{ Marital status } \\
\hline Married & $2117(21.2)$ & 1 & 1 & \\
\hline Single & 7857 (78.8) & $0.9(0.6-1.4)$ & $1.2(0.7-1.9)$ & 0.58 \\
\hline \multicolumn{5}{|l|}{ Education } \\
\hline$\leqq$ senior & $5950(59.7)$ & 1 & 1 & \\
\hline$\geqq$ junior college & 3204 (32.1) & $0.6(0.4-0.8)$ & $0.6(0.4-0.9)$ & 0.01 \\
\hline unknown & $820(8.2)$ & $0.4(0.1-1.0)$ & $0.5(0.2-1.3)$ & 0.15 \\
\hline Suicide attempt (E950-E959) & $186(1.9)$ & $7.1(4.1-12.3)$ & $6.1(3.5-10.8)$ & $<0.001$ \\
\hline No. of physical comorbidities* & $2718(27.3)$ & $1.0(0.6-1.4)$ & $0.8(0.5-1.2)$ & 0.24 \\
\hline No. of pre-existing psychiatric comorbidities\# & $5664(56.8)$ & $2.5(1.7-3.8)$ & $2.4(1.6-3.7)$ & $<0.001$ \\
\hline No. of concurrent psychiatric comorbidities\# & $4624(46.4)$ & $0.8(0.6-1.2)$ & $1.0(0.7-1.5)$ & 0.88 \\
\hline No. of subsequent psychiatric comorbidities\# & $1094(11.0)$ & $0.8(0.5-1.4)$ & $0.8(0.4-1.3)$ & 0.34 \\
\hline \multicolumn{5}{|c|}{$\begin{array}{l}\text { *including: Diabetes mellitus: ICD9_250; Hypertensive Disease: ICD9_401-405; Lipoid metabolism: ICD9_272; Cancer: ICD9_140-208; Cardio Vascular } \\
\text { Disease: ICD9_390-398, 410-414, 420-429; Cerebral Vascular Accident: ICD9_430-438. }\end{array}$} \\
\hline
\end{tabular}

\begin{tabular}{|c|c|c|c|c|}
\hline \multirow[b]{2}{*}{ Variable } & \multicolumn{4}{|c|}{ ED patient $(n=9974)$} \\
\hline & n (\%) & HR $(95 \% \mathrm{Cl})$ & aHR (95\% CI) & $P$ value \\
\hline $\begin{array}{l}\text { No. of six pre-existing psychiatric } \\
\text { comorbidities }\end{array}$ & $5664(56.8)$ & $2.5(1.7-3.8)$ & $2.4(1.6-3.7)$ & $<0.001$ \\
\hline Major depressive disorder & $4285(43.0)$ & $2.5(1.7-3.6)$ & $1.8(1.2-2.7)$ & 0.005 \\
\hline Bipolar disorder & $952(9.5)$ & $2.2(1.3-3.5)$ & $1.0(0.6-1.8)$ & 0.89 \\
\hline Schizophrenia & $326(3.3)$ & $2.7(1.4-5.2)$ & $1.9(1.0-1.8)$ & 0.06 \\
\hline Substance use disorder & $471(4.7)$ & $5.0(3.1-8.1)$ & $2.6(1.5-4.5)$ & 0.001 \\
\hline Anxiety & $3488(35.0)$ & $2.1(1.5-3.0)$ & $1.5(1.0-2.2)$ & 0.06 \\
\hline Personality Disorder & $422(4.2)$ & $3.0(1.7-5.4)$ & $1.2(0.6-2.3)$ & 0.57 \\
\hline Number of comorbidities & $4310(43.2)$ & & & \\
\hline 1 comorbidity & $2767(27.7)$ & $1.6(1.0-2.6)$ & $1.7(1.0-2.7)$ & 0.05 \\
\hline 2 comorbidities & $1899(19.0)$ & $2.9(1.8-4.7)$ & $2.9(1.7-4.8)$ & $<0.001$ \\
\hline 3 comorbidities & $697(7.0)$ & $3.7(2.0-6.9)$ & $3.6(1.8-6.9)$ & $<0.001$ \\
\hline 4 or more comorbidities & $226(2.3)$ & $8.2(4.3-15.6)$ & $6.3(3.1-12.8)$ & $<0.001$ \\
\hline
\end{tabular}

lifetime prevalence at least one mood disorder varies considerably, from $24.1-90 \%$ with $\mathrm{BN}$, while it was $31-88.9 \%$ for AN. Furthermore, patients with ED have a higher prevalence of concurrent mood disorders than the general population. Tseng et al. [27] also conducted a study on psychiatric patients and found that patients with ED had a high percentage of comorbid depressive symptoms (41.3-66.7\%), followed by bipolar disorders (16.7-49.3\%). They demonstrated that patients with ED and comorbid bipolar disorders showed an increased risk of weight dysregulation, impulsive behavior, and suicides. Additionally, alcohol abuse, selfharm, and illicit drugs use were common in women with bulimia and binge eating disorders [28]. Novelty seeking, self-directedness and impulsivity were proposed to be associated with 
both the pathology of substance use and bulimia [29]. Moreover, the prior substance use might complicate the treatment of eating disorders [30]. Those findings provided the evidence and were consistent with our finding that either history of major depression or prior substance use might also be associated with the suicidal death among the patients with ED.

Our investigation also found that ED with at least one pre-existing psychiatric comorbidity had higher risk of suicidal deaths, compared with patients without comorbidities. In addition, patients were more suicide risks as the number of pre-existing psychiatric comorbidities increased. In recent years, Bühren et al. [31] demonstrated the binge-purging subtype and the severity of eating disorder were associated with the presence of suicidal ideation. However, to our knowledge, our study was the first one showed that the preexisting psychiatric comorbidities including major depressive disorders or substance use disorders but not concurrent or subsequent psychiatric comorbidities were associated with the suicidal death of patients with ED. Future studies were warranted to investigate the role of ED in the development of suicidal death of the patients with major depressive disorder and/or substance use disorder. Our results implicated that it is critical for the identification and intervention of the pre-existing psychiatric comorbidities among the patients with ED since there was addictive effect regarding the numbers of psychiatric comorbidities. On the other hand, we did not find the impact of comorbidities of anxiety disorders, personality disorders, schizophrenia in the suicidal death among the patient with ED although the impulsivity, and interpersonal deficits might explain the pathology of obsessive-compulsive disorder [32,33], personality disorders [34-36], schizophrenia [37,38] and eating disorders.

Some limitations of the study must be noted. First, to increase the specificity of the ED diagnosis, only patients with $\geq 4$ diagnoses by psychiatrists was included in the study. Second, the data from the National Health Insurance Database lacked some clinical information, such as height, weight, family history, blood examination, and psychological behavioral therapy. This could have limited the comprehensive comparison of similarities and differences between patients with different types of ED. Third, data on the level of education of the patient cohort in the current study were obtained based on the enrollment status. Since this data does not require mandatory recording, part of the data could be missing; thus, its accuracy requires further confirmation.

In fact, apart from psychiatric disorders affecting the risk of suicides, other factors such as genetics $[39,40]$, society $[41,42]$, family [43], and cognitive behavior [44,45] may have also increased the risk of suicidal thoughts and depressive symptoms. This study only carried out a preliminary investigation based on databases. Future studies focusing on one type of ED, including a collection of medical examinations and survey questionnaires, could be conducted to further validate our findings.

\section{Conclusions}

The risk of suicide was linked with psychiatric comorbidity in patients with $\mathrm{ED}$, particularly those with pre-existing major depression and/ or substance use disorders. Future longitudinal studies were warranted to validate the preliminary results from the investigation of databases.

\section{Acknowledgments}

The data from this study were provided by the data science center of the Taiwan Ministry of Health and Welfare. The explanation and conclusions do not represent the views of the Ministry of Health and Welfare. This study was supported by the Tri-Service General Hospital (TSGH-C105-139). CLY, JCC and $M K Y$ were responsible for study concept and design. SCW acquired the data. CLY, JCC and CBY analyzed and interpreted the data. CLY and JCC drafted the manuscript. MKY and CBY critically revised the manuscript for important intellectual content. MKY provided administrative, technical or material support. All authors contributed to the manuscript and approved the final version.

This study also approved by the Institutional Review Board of the Tri-Service General Hospital (reference number 1-104-05-048) and was conducted in accordance with the Helsinki Declaration. Informed consent from patients was not required for this study, as they were not asked to follow rules of behavior. Patient data were coded and anonymity of patients was guaranteed.

\section{Conflicts of Interest}

None of the authors report any conflicts of interest. 


\section{References}

1. Smink FR, van Hoeken D, Hoek HW. Epidemiology of eating disorders: incidence, prevalence and mortality rates. Curr. Psychiatry. Rep 14(4), 406-414 (2012).

2. Pike KM, Dunne PE. The rise of eating disorders in Asia: a review. J. Eat. Disord 3, 33 (2015).

3. Johnson JG, Cohen P, Kotler L, et al. Psychiatric disorders associated with risk for the development of eating disorders during adolescence and early adulthood. J. Consult. Clin. Psychol 70(5), 1119-1128 (2002).

4. Bulik CM, Sullivan PF, Fear JL, et al. Eating disorders and antecedent anxiety disorders: a controlled study. Acta. Psychiatr. Scand 96(2), 101-107 (1997).

5. Stice E, Hayward C, Cameron RP, et al. Body-image and eating disturbances predict onset of depression among female adolescents: a longitudinal study. J. Abnorm. Psychol 109(3), 438-444 (2000).

6. Thompson-Brenner H, Eddy KT, Franko DL, et al. Personality pathology and substance abuse in eating disorders: a longitudinal study. Int. J. Eat. Disord 41 (3), 203-208 (2008).

7. Chen EY, McCloskey MS, Michelson S, et al. Characterizing eating disorders in a personality disorders sample. Psychiatry. Res 185(3), 427-432 (2011).

8. Favaro A, Santonastaso P. Suicidality in eating disorders: clinical and psychological correlates. Acta. Psychiatr. Scand 95(6), 508514 (1997).

9. Franko DL, Keel PK. Suicidality in eating disorders: Occurrence, correlates, and clinical implications. Clin. Psychol. Rev 26(6), 769-782 (2006).

10. Bodell LP, Joiner TE, Keel PK. Comorbidityindependent risk for suicidality increases with bulimia nervosa but not with anorexia nervosa. J. Psychiatr. Res 47(5), 617-621 (2013).

11. Milos G, Spindler A, Hepp U, et al. Suicide attempts and suicidal ideation: links with psychiatric comorbidity in eating disorder subjects. Gen. Hosp. Psychiatry 26(2), 129135 (2004).

12. Arcelus J, Mitchell AJ, Wales J, et al. Mortality rates in patients with anorexia nervosa and other eating disorders. A metaanalysis of 36 studies. Arch. Gen. Psychiatry 68(7), 724-731 (2011).

13. Tseng MM, Fang D, Lee MB, et al. Twophase survey of eating disorders in gifted dance and non-dance high-school students in Taiwan. Psychol. Med 37(8), 1085-1096 (2007).

14. Liu CY, Tseng MC, Chang CH, et al.
Comorbid psychiatric diagnosis and psychological correlates of eating disorders in dance students. J. Formos. Med. Assoc 115(2), 113-120 (2016).

15. Lee S, Lee AM. Disordered eating in three communities of China: a comparative study of female high school students in hong kong, Shenzhen, and rural hunan. Int. J. Eat. Disord 27(3), 317-327 (2000).

16. Nakamura K, Yamamoto M, Yamazaki O, et al. Prevalence of anorexia nervosa and bulimia nervosa in a geographically defined area in Japan. Int. J. Eat. Disord 28(2), 173180 (2000).

17. Lee S, Chiu HF, Chen CN. Anorexia nervosa in Hong Kong. Why not more in Chinese? Br. J. Psychiatry 154(1), 683-688 (1989).

18. Buhrich N. Frequency of presentation of anorexia nervosa in Malaysia. Aust. N.Z. J. Psychiatry 15(2), 153-155 (1981).

19. Wu CS, Lai MS, Gau SS, et al. Concordance between patient self-reports and claims data on clinical diagnoses, medication use, and health system utilization in Taiwan. PLoS. One 9(12), e112257 (2014).

20. Garfinkel PE, Lin E, Goering P, et al. Bulimia nervosa in a Canadian community sample: prevalence and comparison of subgroups. Am. J. Psychiatry 152(7), 1052-1058 (1995).

21. Garfinkel PE, Lin E, Goering P, et al. Should amenorrhoea be necessary for the diagnosis of anorexia nervosa? Evidence from a Canadian community sample. Br. J. Psychiatry 168(4), 500-506 (1996).

22. Striegel-Moore RH, Leslie D, Petrill SA, et al. One-year use and cost of inpatient and outpatient services among female and male patients with an eating disorder: evidence from a national database of health insurance claims. Int. J. Eat. Disord 27(4), 381-389 (2000).

23. Sharan $P$, Sundar AS. Eating disorders in women. Indian. J. Psychiatry 57(Suppl 2), S286-295 (2015).

24. Sadock BJ, Sadock VA, Ruiz P, et al. Kaplan \& Sadock's comprehensive textbook of psychiatry. Philadelphia: Wolters Kluwer Health/Lippincott Williams \& Wilkins. (2009).

25. Chandra PS CS, Jagdeesh AN. Textbook of Postgraduate Psychiatry. In: Vyas JN, Ahuja N, editors. 2nd ed. New Delhi: Jaypee Brothers Publishers. pp. 357-368. (1999).

26. Godart NT, Perdereau F, Rein Z, et al. Comorbidity studies of eating disorders and mood disorders. Critical review of the literature. J. Affect. Disord 97(1-3), 37-49 (2007).

27. Tseng MC, Chang $\mathrm{CH}$, Chen $\mathrm{KY}$, et al Prevalence and correlates of bipolar disorders in patients with eating disorders.
J. Affect. Disord 190, 599-606 (2016).

28. Dohm FA, Striegel-Moore RH, Wilfley DE, et al. Self-harm and substance use in a community sample of Black and White women with binge eating disorder or bulimia nervosa. Int. J. Eat. Disord 32(4), 389400 (2002).

29. Del Pino-Gutierrez A, Jimenez-Murcia S, Fernandez-Aranda F, et al. The relevance of personality traits in impulsivity-related disorders: From substance use disorders and gambling disorder to bulimia nervosa. J. Behav. Addict 6(3), 396-405 (2017).

30. Strasser TJ, Pike KM, Walsh BT. The impact of prior substance abuse on treatment outcome for bulimia nervosa. Addict. Behav 17(4), 387-395 (1992).

31. Bühren K, Schwarte R, Fluck F, et al. Comorbid psychiatric disorders in female adolescents with first-onset anorexia nervosa. Eur. Eat. Disord. Rev 22(1), 39-44 (2014).

32. Belloch A, Roncero M, Perpina C. Obsessional and Eating Disorder-related Intrusive Thoughts: Differences and Similarities Within and Between Individuals Vulnerable to OCD or to EDs. Eur. Eat. Disord. Rev 24(6), 446-454 (2016).

33. Micali N, Hilton $\mathrm{K}$, Nakatani $\mathrm{E}$, et al. Is childhood OCD a risk factor for eating disorders later in life? A longitudinal study. Psychol. Med 41(12), 2507-2513 (2011).

34. Davis C, Mackew L, Levitan RD, et al. Binge Eating Disorder (BED) in Relation to Addictive Behaviors and Personality Risk Factors. Front. Psychol 8(1), 579 (2017).

35. Mason TB, Lavender JM, Wonderlich SA, et al. The role of interpersonal personality traits and reassurance seeking in eating disorder symptoms and depressive symptoms among women with bulimia nervosa. Compr. Psychiatry 68(1), 165-171 (2016).

36. Munch AL, Hunger C, Schweitzer J. An investigation of the mediating role of personality and family functioning in the association between attachment styles and eating disorder status. BMC. Psychol 4(1), 36 (2016).

37. Kouidrat Y, Amad A, Lalau JD, et al. Eating disorders in schizophrenia: implications for research and management. Schizophr. Res. Treatment 2014, 791573 (2014).

38. Lyketsos GC, Paterakis $P$, Beis $A$, et al. Eating disorders in schizophrenia. Br. J. Psychiatry 146(1), 255-261 (1985).

39. Treasure J, Holland A. Genetic vulnerability to eating disorders: evidence from twin and family studies. H Remschmidt, MH Schmidt (Eds.), Child and youth psychiatry: European perspectives. Hogrefe and Huber, New 


\section{Research Chin-Bin Yeh}

York, . pp. 59-68 (1989).

40. Strober M, Freeman R, Lampert C, et al. Controlled family study of anorexia nervosa and bulimia nervosa: evidence of shared liability and transmission of partial syndromes. Am. J. Psychiatry 157(3), 393401 (2000).

41. Wertheim EH, Paxton SJ, Schutz HK, et al. Why do adolescent girls watch their weight? An interview study examining sociocultural pressures to be thin. J.
Psychosom. Res 42(4), 345-355 (1997).

42. Akan GE, Grilo CM. Sociocultural influences on eating attitudes and behaviors, body image, and psychological functioning: a comparison of African-American, AsianAmerican, and Caucasian college women. Int. J. Eat. Disord 18(2), 181-187 (1995).

43. McCabe MP, Ricciardelli LA. A prospective study of pressures from parents, peers, and the media on extreme weight change behaviors among adolescent boys and girls.
Behav. Res. Ther 43(5), 653-668 (2005).

44. Vander Wal JS. Eating and body image concerns among average-weight and obese African American and Hispanic girls. Eat. Behav 5(2), 181-187 (2004).

45. Ruffolo JS, Phillips KA, Menard W, et al. Comorbidity of body dysmorphic disorder and eating disorders: severity of psychopathology and body image disturbance. Int. J. Eat. Disord 39(1), 11-19 (2006). 